\title{
Vecteurs au lycée : difficile articulation entre mathématiques
} et physique

\section{Vectors at the lycée: uneasy articulation between mathematics and physics \\ Vectores en el liceo: difícil articulación entre matemáticas y física}

\section{Cissé Ba}

Volume 14, numéro 1, 2011

Les approches intégratives : quelles spécificités en enseignement des mathématiques, sciences et technologies?

URI : https://id.erudit.org/iderudit/1008844ar

DOI : https://doi.org/10.7202/1008844ar

Aller au sommaire du numéro

Éditeur(s)

Faculté d'éducation, Université de Sherbrooke

ISSN

1911-8805 (numérique)

Découvrir la revue

Citer cet article

Ba, C. (2011). Vecteurs au lycée : difficile articulation entre mathématiques et physique. Nouveaux cahiers de la recherche en éducation, 14(1), 71-83. https://doi.org/10.7202/1008844ar
Résumé de l'article

Ce texte issu de notre doctorat présente une recherche sur les interactions entre mathématiques et physique à propos des vecteurs et des grandeurs physiques vectorielles. Nous présentons tout d'abord une synthèse de l'évolution de l'enseignement de ces deux notions au cours du XX $\mathrm{X}^{\mathrm{e}}$ siècle. Puis, sur la base d'une analyse des programmes actuels et de quelques manuels, nous évaluons comment cette interaction est actuellement gérée en France. Enfin, nous présentons brièvement les résultats d'un questionnaire que nous avons réalisé et distribué à des enseignants des deux disciplines en France et au Sénégal. 


\title{
Vecteurs au lycée : difficile articulation entre mathématiques et physique
}

\author{
Cissé Ba \\ Université Cheikh Anta Diop
}

\section{Vecteurs au lycée : difficile articulation entre mathématiques et physique}

\section{Résumé}

Ce texte issu de notre doctorat présente une recherche sur les interactions entre mathématiques et physique à propos des vecteurs et des grandeurs physiques vectorielles. Nous présentons tout d'abord une synthèse de l'évolution de l'enseignement de ces deux notions au cours du XX $\mathrm{X}^{\mathrm{e}}$ siècle. Puis, sur la base d'une analyse des programmes actuels et de quelques manuels, nous évaluons comment cette interaction est actuellement gérée en France. Enfin, nous présentons brièvement les résultats d'un questionnaire que nous avons réalisé et distribué à des enseignants des deux disciplines en France et au Sénégal.

Mots-clés : vecteur, enseignement des mathématiques, enseignement de la physique.

\section{Vectors at the lycée: uneasy articulation between mathematics and physics}

\section{Abstract}

Based on the author's doctoral dissertation, the article introduces a research on interactions between mathematics and physics about vectors and physical vector quantities. First, the article summarizes the evolution in the teaching of these two notions during the 20th Century. Then, based on the analysis of actual programs and teaching manuals, the article presents an assessment of the management of these interactions in France as well as the results of a questionnaire that was answered by teachers of these two disciplines in France and Senegal.

Keywords: vector, mathematics teaching, physics teaching.

\section{Vectores en el liceo: difícil articulación entre matemáticas y física}

\section{Resumen}

El presente texto, fruto de nuestro trabajo doctoral, presenta una investigación sobre las interacciones entre matemáticas y física en torno a los vectores y de las magnitudes físicas vectoriales. En primer lugar presentamos una síntesis de la evolución de la enseñanza de estas dos nociones durante el siglo XX. Luego, en base a un análisis de los programas actuales y de algunos libros, evaluamos cómo se maneja esta interacción en Francia en la actualidad. Por terminar, presentamos brevemente los resultados de un cuestionario que administramos a unos docentes de las dos disciplinas en Francia y en Senegal.

Palabras clave: vector, enseñanza de las matemáticas, enseñanza de la física. 


\section{Introduction}

Depuis longtemps, les mathématiques et les sciences physiques ont entretenu des rapports privilégiés, que ce soit dans la constitution des savoirs savants ou dans leur enseignement. Néanmoins, ces rapports ont subi d'importantes mutations, qui ont marqué les pratiques d'enseignement dans les deux disciplines.

Comme l'a montré Artaud (1999), aux XVII ${ }^{\mathrm{e}}$ et XVIII ${ }^{\mathrm{e}}$ siècles, le découpage disciplinaire assurait l'existence de mathématiques dites "mixtes" englobant, entre autres, la mécanique, qui correspondaient à une réelle visibilité sociale des mathématiques. Selon ce découpage, les liens avec la physique étaient non seulement forts, mais dépassaient le simple recours utilitariste aux mathématiques par les autres disciplines, puisqu'il s’agissait «moins de mettre des mathématiques dans la physique que d'amener les questions de physique dans le champ des mathématiques» (Ibid., p. 26). Dans le courant des $\mathrm{XIX}^{\mathrm{e}}$ et $\mathrm{XX}^{\mathrm{e}}$ siècles, les mathématiques ont subi un phénomène “d'implicitation”, amplifié par un nouveau découpage en mathématiques pures et appliquées.

$[\ldots]$ là où les mathématiques mixtes partaient de questions non mathématiques pour les mathématiser, les mathématiques appliquées mettent en avant les théories mathématiques et ne font apparaître les questions auxquelles elles répondent qu'occasionnellement, en fin de parcours. (Ibid., p. 33)

Dans la période des années 1970, caractérisée par le courant dit des “mathématiques modernes", l'enseignement des mathématiques ne prenait quasiment pas en compte leurs liens avec les sciences physiques, alors que l'enseignement de ces dernières s'est fortement mathématisé.

Dans cette évolution tracée ici à grands traits, on voit que les rapports entre les enseignements de mathématiques et de sciences physiques ont abouti à une distanciation importante et à un rapport de hiérarchie peu favorable à une collaboration égalitaire. Depuis les années 1980, les réformes successives, qui s'inscrivent dans une réaction au radicalisme des mathématiques modernes, ont prôné un rapprochement des deux disciplines. Ce phénomène s'inscrit dans un mouvement général, d'une part, de la nécessité de redonner aux mathématiques une base "concrète" et utilitaire - partir de la contextualisation des savoirs mathématiques - et d'autre part, de lutter contre le repliement disciplinaire, pour la promotion de l'interdisciplinarité.

On trouve ainsi, dans divers textes officiels récents, des injonctions comme:

Dans cette perspective, l'enseignement des mathématiques est aussi à relier à celui des autres disciplines sous deux aspects principaux: étude des situations issues de ces disciplines; organisation concertée des activités d'enseignement. (Ministère de l'Éducation nationale, 1990)

D'une manière générale, l'introduction d'une notion par la théorie est vivement déconseillée. Il sera souvent fait appel à l'expérience scientifique de l'élève et aux problèmes des autres disciplines pour décloisonner l'enseignement des mathématiques. (Ministère de l'Éducation, 2006)

Ou encore,

La conception des mathématiques comme une science pure, jeu autonome dans le monde des idées, est peut-être confortable pour quelques-uns. Mais cette conception 
gomme les interactions pourtant si fécondes, entre les mathématiques et les autres sciences. Plutôt que de se limiter à des vœux pieux, les programmes devront proposer des exemples précis d'applications des mathématiques. (Groupe de réflexion InterAssociations en Mathématiques [GRIAM], 1998)

Dans ces trois citations, on voit bien la volonté de faire sortir les mathématiques de leur tour d'ivoire en redécouvrant, entre autres, les vertus de leur application aux autres disciplines, dont la physique au premier titre, mais aussi, la difficulté ("vœux pieux") à proposer dans les programmes de réelles activités conduisant à une pertinente modélisation mathématique.

D'autant que, dans le même temps, la noosphère ${ }^{1}$ des sciences physiques, en réaction à "l'impérialisme" des mathématiques, a promu un enseignement qui met de l'avant le rôle de l'expérience et les aspects qualitatifs, parallèlement à moins de mathématisation.

L'hypothèse à la base de notre travail est que les changements de programmes, même importants, ne peuvent suffire à eux seuls à combattre le repliement disciplinaire. Non seulement un accompagnement au plan de la formation (initiale et continue) des enseignants des deux disciplines est nécessaire, mais aussi, et peut-être avant tout, il faut arriver à annihiler les effets pervers d'une culture installée de l'isolationnisme, qui conduit à refuser toute incursion de la problématique de l'autre discipline dans le pré carré de la sienne. C'est ce réseau de questions que nous avons abordé dans notre thèse ${ }^{2}$, en nous centrant sur les concepts de vecteurs et de grandeurs physiques vectorielles d'une part et de translation et mouvement de translation d'autre part. Dans cet article, nous ne toucherons que le premier des deux thèmes et n'aborderons pas les aspects liés à la translation (Ba, 2007 et $\mathrm{Ba}$ et Dorier, 2007).

\section{Aperçu de l'histoire de l'enseignement du vecteur}

Même si l'on retrouve des traces du parallélogramme de forces dès l'antiquité, l'origine du vecteur est à chercher dans des périodes beaucoup plus récentes. La critique de Leibniz de la géométrie de Descartes (Crowe, 1967), qui prônait la recherche d'une caractéristique purement géométrique s'appliquant aux positions comme l'algèbre s'applique aux grandeurs, est restée lettre morte pendant plus d'un siècle. C'est vraiment avec l'interprétation géométrique des quantités imaginaires et le désir de généralisation à l'espace, que le concept de vecteur se fait jour dans le courant du $\mathrm{XIX}^{\mathrm{e}}$ siècle, à la croisée de l'algèbre et de la géométrie, puis dans les applications à la physique (Crowe, 1967; Flament,1997 et 2003). De même, les liens qui ont uni la genèse du calcul vectoriel et l'élaboration de l'algèbre linéaire sont aussi plus complexes et plus ténus qu'ils n'en ont l'air (Dorier, 1997).

Notre propos n'étant pas ici de retracer l'histoire des vecteurs, nous renvoyons le lecteur intéressé aux ouvrages cités ci-dessus. Nous nous intéressons plutôt à l'histoire de l'enseignement des vecteurs en France, depuis leur timide apparition dans les programmes des classes du secondaire

1 Chevallard (1985) a emprunté à Teilhard de Chardin le terme "noosphère" pour désigner l'ensemble des lieux, des instances où se passent les échanges entre le système d'enseignement et son environnement: cette noosphère peut regrouper des membres d'associations de spécialistes, de commissions, permanentes ou éphémères, de réflexion sur l'enseignement, etc.

2 Étude épistémologique et didactique de l'utilisation des vecteurs en physique et en mathématiques. Thèse de doctorat sous la direction de Jean-Luc Dorier. 
à la fin du XIX ${ }^{\mathrm{e}}$ siècle jusqu'à nos jours. Au-delà de l'intérêt historique, nous voulons ainsi éclairer un domaine de l'éducation mathématique qui ne cesse de rétrécir au fur et à mesure des réformes récentes, et dont le lien avec l'enseignement de la physique, s'il paraît naturel aux deux parties, semble néanmoins ne pas pouvoir réellement servir d'appui efficace pour les enseignants de l'une et l'autre discipline.

Du point de vue théorique de l'analyse, nous nous situons dans une perspective écologique, c'est-à-dire que nous identifions l'évolution de l'habitat et des niches des vecteurs, selon les termes définis par Chevallard (1994) dans son approche de l'écologie didactique des savoirs:

Les écologistes distinguent, s'agissant d'un organisme, son habitat et sa niche. Pour le dire en un langage volontairement anthropomorphe, l'habitat, c'est en quelque sorte l'adresse, le lieu de résidence de l'organisme. La niche, ce sont les fonctions que l'organisme y remplit : c'est en quelque façon la profession qu'il y exerce. (Ibid., p. 142).

À la suite de Chevallard, Artaud (1997) montre alors comment un objet émerge et peut vivre dans un écosystème didactique.

Pour qu'un objet $\mathrm{O}$ émerge dans un écosystème didactique, il est nécessaire qu'existe un milieu pour cet objet, c'est-à-dire un ensemble d'objets connus (au sens où il existe un rapport institutionnel non problématique) avec lesquels $\mathrm{O}$ viendra se mettre en interrelation. Cette condition est à mettre en rapport avec une condition citée plus haut, la loi du tout structuré, dont je rappelle l'énoncé: un objet mathématique ne peut exister seul; il doit venir prendre place dans une organisation mathématique, organisation qu'il faut faire exister. La nécessité qu'existe un milieu dit alors que cette émergence d'une organisation mathématique ne peut se faire ex nihilo. Il faut prendre appui sur des organisations, mathématiques ou non mathématiques, déjà existantes. (Ibid., p. 124).

L'analyse écologique permettra donc de mettre au jour un réseau de conditions et de contraintes qui vont déterminer la place que peut occuper l'objet vecteur et son évolution au cours des changements de programmes en prenant en compte le fonctionnement global des institutions scolaires où il intervient.

Dans ce qui suit, nous ne donnons qu'une synthèse de ces analyses sous la forme d'un tableau, dans lequel nous distinguons cinq grandes périodes de 1852 à nos jours (pour une analyse plus complète, voir Ba, 2007, Ba et Dorier, 2006 et Dorier, 2000). 
Tableau 1

Tableau récapitulatif de l'enseignement du vecteur

\begin{tabular}{|c|c|c|}
\hline Périodes & Dates et faits marquants & Habitats et niches \\
\hline \multirow{3}{*}{$\begin{array}{l}\text { Les débuts } \\
1852-925\end{array}$} & $\begin{array}{l}1852 \\
\text { Première référence au mot } \\
\text { "vecteur" }\end{array}$ & $\begin{array}{l}\text { Rayon vecteur dans les programmes de mathématiques } \\
\text { du secondaire. } \\
\text { Allusion au parallélogramme de forces et à la } \\
\text { composition des forces concourantes ou parallèles. } \\
\text { Mais absence de lien avec la notion de vecteur. }\end{array}$ \\
\hline & $\begin{array}{c}1902 \\
\text { Première apparition du vecteur } \\
\text { en première }\end{array}$ & $\begin{array}{l}\text { Habitat paraphysique (Mécanique - Cinématique) } \\
\text { Niche: représentation de grandeurs physiques (force et } \\
\text { vitesse). }\end{array}$ \\
\hline & $\begin{array}{c}1905 \\
\begin{array}{c}\text { Modification et allégement de } \\
\text { programme }\end{array} \\
\end{array}$ & $\begin{array}{l}\text { Habitat: Géométrie en terminale } \\
\text { cependant pas de changement véritable de niche } \\
\text { Adaptation purement didactique; }\end{array}$ \\
\hline \multirow{3}{*}{$\begin{array}{c}\text { Une évolution lente } \\
1937-1957\end{array}$} & $\begin{array}{l}1925 \\
\text { Nouvel habitat (arithmétique) } \\
\text { potentiel en troisième } \\
\text { Légère modification en terminale } \\
\text { (géométrie -> trigonométrie) } \\
\text { Pas de lien entre les deux habitats }\end{array}$ & $\begin{array}{l}\text { Troisième } \\
\text { Habitat (potentiel) : arithmétique } \\
\text { Niche: Représentation des grandeurs mesurables } \\
\text { susceptibles de sens. } \\
\text { Terminale } \\
\text { Habitat: trigonométrie } \\
\text { L'intervention des vecteurs en cinématique est plus } \\
\text { précise. } \\
\text { Ainsi, le statut géométrique des vecteurs se renforce et } \\
\text { leur niche dans cet habitat se consolide dans le rapport } \\
\text { à la trigonométrie. }\end{array}$ \\
\hline & $\begin{array}{l}\text { 1937-1938 } \\
\text { Maintien des deux habitats } \\
\text { précédents } \\
\text { Nouvel habitat (algébrique) }\end{array}$ & $\begin{array}{l}\text { Les habitats et les niches ne changent guère. } \\
\text { l'habitat algébrique se renforce (importance de la } \\
\text { multiplication par un scalaire) tout en se limitant à la } \\
\text { dimension } 1 . \\
\text { L'habitat trigonométrique descend en première. }\end{array}$ \\
\hline & $\begin{array}{l}1947 \\
\text { Pont entre les deux habitats } \\
\text { géométrie et algèbre }\end{array}$ & $\begin{array}{l}\text { Peu de modification. } \\
\text { L'habitat trigonométrie s'élargit à toute la géométrie. } \\
\text { Pont entre les deux habitats (géométrie, algèbre), par } \\
\text { le théorème de Thalès qui associe géométrie et mesure } \\
\text { algébrique. Importance de la multiplication par un } \\
\text { scalaire et de l'équipollence. }\end{array}$ \\
\hline $1957-1967$ & Renforcement & $\begin{array}{l}\text { Habitats et niches restent inchangés alors que l'outil } \\
\text { vectoriel s'impose de plus en plus (apparition } \\
\text { en physique en classe de première à propos } \\
\text { d'électromagnétisme). }\end{array}$ \\
\hline $\begin{array}{l}\text { 1968-1985 } \\
\text { Réforme des mathé- } \\
\text { matiques modernes }\end{array}$ & Le vecteur envahit la géométrie & $\begin{array}{l}\text { Habitat: géométrie } \\
\text { Niche: fondements de la géométrie, préparation à } \\
\text { l'algèbre linéaire. }\end{array}$ \\
\hline $\begin{array}{c}\text { 1985-2006 } \\
\text { La contre-réforme }\end{array}$ & $\begin{array}{l}\text { Le vecteur est réduit au rang } \\
\text { d'outil et occupe une place } \\
\text { réduite en géométrie }\end{array}$ & $\begin{array}{l}\text { Habitat (réduit): géométrie } \\
\text { Niche: illustration de la physique et outil performant } \\
\text { pour faire de la géométrie. } \\
\text { La référence à l'algèbre (que ce soit l'algèbre linéaire } \\
\text { ou les grandeurs orientées, comme on l'a vu dans } \\
\text { la niche arithmétique des années } 1940 \text { à 1960) a } \\
\text { complètement disparu. }\end{array}$ \\
\hline
\end{tabular}

3 Niche arithmétique: Sans être explicitement au programme, les vecteurs apparaissent en classe de troisième, pour la représentation des nombres relatifs comme "notions concrètes sur les nombres positifs et négatifs" en arithmétique. 
De façon générale, il ressort de ces analyses que l'évolution des programmes n'a cessé de séparer les habitats physique et mathématique du vecteur, favorisant ainsi le cloisonnement disciplinaire. Depuis quelques années, on semble vouloir revenir à un lien plus étroit des vecteurs avec les forces ou les vitesses, mais cette injonction s'appuie-t-elle sur une base réaliste?

De notre analyse historique de l'évolution de l'enseignement du vecteur, nous retiendrons trois points propres à éclairer la situation actuelle de l'enseignement des vecteurs et des liens possibles avec la physique:

- Malgré le rejet de la réforme des mathématiques modernes, le modèle de l'algèbre linéaire, s'il a disparu officiellement des programmes du secondaire, continue de marquer l'organisation mathématique autour du vecteur. L'importance accordée à la multiplication par un scalaire en classe de seconde en atteste.

- Par ailleurs, la niche "outil performant pour la géométrie" a elle aussi du mal à fonctionner (Le Thi Hoai, 1997; Bittar, 1998; Pressiat, 1999). Il est en effet difficile de trouver un problème de géométrie initialement posé sans vecteur, où la modélisation vectorielle contribue à simplifier sa résolution au point d'y faire apparaître l'outil vectoriel comme significativement performant. On a vu en effet, à travers l'évolution des programmes (et l'analyse historique le confirme) que l'habitat géométrique n'est pas si naturel qu'il y paraît pour les vecteurs. Pour une part importante, le vecteur géométrique est une création didactique qui a permis à un moment donné de résoudre un problème idéologique (enseigner dans le secondaire que ce qui prépare au supérieur: plaquer le modèle de l'espace euclidien normé pour l'introduction du vecteur au collège dans la perspective de l'enseignement de l'algèbre linéaire, et même plus loin de l'analyse fonctionnelle) et pratique dans l'organisation du savoir enseigné (Dorier, 2000).

- Reste la niche "outil pour la physique", mais elle paraît aussi difficile à faire vivre. En effet, peu de situations physiques sont utilisables en troisième ou même en seconde, dans lesquelles le formalisme vectoriel est vraiment pertinent.

Du point de vue de l'enseignement des sciences physiques, l'introduction du vecteur pour modéliser les grandeurs physiques est relativement récente et a fait l'objet de nombreux débats (Ba, 2007).

\section{3. Éléments d'analyse institutionnelle}

Dans ce paragraphe, nous donnons un aperçu partiel du rapport institutionnel actuel aux objets vecteurs et grandeurs physiques vectorielles en France et au Sénégal à la fin du collège et au début du lycée. Nous renvoyons le lecteur à $\mathrm{Ba}$ (2007) pour une étude détaillée.

En France, les références à la physique dans la partie des programmes de mathématiques sur les vecteurs ont quasiment disparu depuis 2000 après une brève apparition dans les années 1980-1990. Au Sénégal, il reste en seconde une courte allusion : «On montrera l'utilité de l'outil vectoriel dans d'autres disciplines ».

Par ailleurs, nous avons analysé sept manuels récents de mathématiques de troisième édités en France et six de seconde. Moins de la moitié (quatre de troisième et seulement deux de seconde) présentent une ou plusieurs situations illustrant les liens entre vecteurs et grandeurs physiques vectorielles, la plupart du temps, à une place secondaire, voire marginale, dans l'organisation des 
manuels. En effet, les quelques collections qui en proposent les renvoient de manière systématique en fin de chapitre sous forme d'exercices corrigés ou de travaux pratiques. Ainsi, il apparaît que le lien entre mathématiques et physique est peu abordé, et encore que de façon assez anecdotique. Examinons toutefois un exemple où ce lien est particulièrement mis en avant.

Dans le manuel de seconde de Lotz (Turner et Misset, 2004), dans le chapitre sur les vecteurs, on trouve, entre autres, l'exercice suivant:

\section{À l'entraînement}

Au cours d'une séance d'entraînement de rugby, afin de travailler la puissance des joueurs, l'exercice suivant est proposé : un des joueurs $\mathrm{J}_{1}$ est retenu à l'aide de deux cordes par deux autres joueurs $\mathrm{J}_{2}$ et $\mathrm{J}_{3}$. Dans chacun des cas ci-dessous, le joueur $\mathrm{J}_{1}$ va-t-il avancer ou reculer?

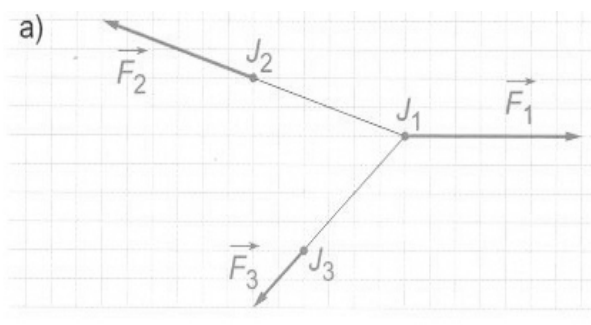

b)

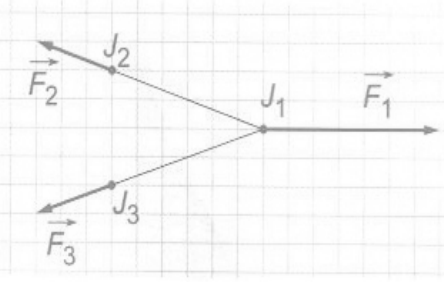

c)

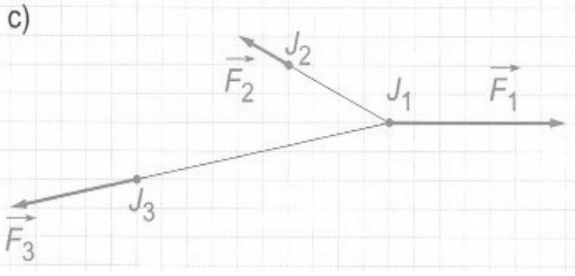

Cet exercice s'apparente effectivement à des connaissances de physique, mais il est remarquable que la modélisation soit considérée comme totalement transparente. Dans le texte on parle de puissance des joueurs, sans la définir, ni dire par quoi elle est représentée. C'est à l'élève de comprendre que c'est dans le dessin, ce qui est représenté par un vecteur. Mais ceci a plus de chance de se faire par un jeu de contrat didactique que par une réflexion enrichissante de l'élève sur l'intérêt des vecteurs pour modéliser de telle situation. Reste ensuite à comprendre que l'on peut (on doit d'ailleurs) ramener les trois vecteurs en un même point ( $\mathrm{J}_{1}$ est le meilleur candidat) et que répondre à la question posée revient à comparer $\overrightarrow{F_{1}}$ à la somme $\overrightarrow{F_{2}}+\overrightarrow{F_{3}}$. Cette tâche nécessite de faire graphiquement la somme de deux vecteurs, ce qui semble bien être le réel enjeu attendu des auteurs. Pourtant, la question de la comparaison aurait pu mener à des questionnements intéressants puisqu'en effet, il est plus difficile de comparer des vecteurs que des scalaires. Mais ici tout est court-circuité par le fait que les trois exemples éludent la question. En effet, dans les trois cas, la somme $\overrightarrow{F_{2}}+\overrightarrow{F_{3}}$ est toujours dans la même direction et de sens opposé par rapport à $\vec{F}_{1}$, ramenant la discussion à la comparaison de grandeurs scalaires positives (bien entendu, les trois exemples correspondent aux trois cas possibles!). Cet exercice est donc une parodie de modélisation, où le seul enjeu véritable, outre qu'il faut savoir décoder les attentes des auteurs, est de construire graphiquement la somme de deux vecteurs et de comparer des longueurs. 
On voit donc que le lien entre mathématiques et physique à propos des vecteurs a beaucoup de difficultés à vivre dans les chapitres sur les vecteurs au lycée. Le rapport institutionnel au vecteur dans la classe de mathématiques ne laisse que peu d'espace pour des situations issues de la physique. Quand elles existent, celles-ci restent subordonnées à un rapport inadéquat à la modélisation et apparaissent comme un prétexte à faire faire des mathématiques; tout ce qui relève de la modélisation étant considéré comme transparent et conduisant soit à des simplifications drastiques, soit à laisser comme seule possibilité à l'élève de deviner les attentes du professeur.

Ce constat peut paraître radical, mais il a aussi des causes profondes qui ne sont pas à mettre seulement sur le dos des auteurs de manuels. En effet, on voit bien que le décalage dans le temps entre les enseignements de mathématiques et de physique rend difficile la vie, dans la classe de mathématiques, de problèmes où un questionnement physique pourrait être à la source d'un réel travail de modélisation de la part des élèves conduisant à une utilisation problématisée des vecteurs. Ce décalage pourrait à la limite se régler par une modification curriculaire. Néanmoins, les situations physiques présentes dans les manuels de mathématiques sont peu réalistes du point de vue de la problématique physique. Ceci dépasse le simple aspect curriculaire et met bien en évidence un aspect plus pernicieux du cloisonnement disciplinaire.

Regardons à présent rapidement ce qu'il en est de l'enseignement de la physique. Les élèves apprennent à utiliser des représentations vectorielles pour les forces et les vitesses, en classe de seconde $\mathrm{S}$ au Sénégal et en première $\mathrm{S}$ en France. Dans les deux cas, ils ont déjà eu une pratique des vecteurs assez importante en classe de mathématiques. Comme pour les mathématiques, nous avons analysé les programmes et des manuels, mais nous ne pouvons rendre compte de nos analyses de façon détaillée ici.

Il ressort essentiellement de notre étude que les objets mathématiques ne sont traités que comme des outils. Les difficultés éventuelles des élèves avec les vecteurs sont ainsi mises sur le compte de déficiences de l'enseignement des mathématiques, sans qu'un questionnement propre à la nature des liens avec les objets physiques puisse être perçu comme un levier intéressant. À titre d'exemple, voici un extrait du manuel de Tomasino (2001) de première S. Ce court encadré, intitulé "point-méthode", se présente comme un rappel de mathématiques et se trouve de fait totalement déconnecté de toute référence à la physique, même si les vecteurs sont notés avec la lettre $F$ (comme force) et non $v$ ou $u$, comme en mathématiques. 
1. Méthode géométrique

\section{Déterminer le vecteur $\vec{F}=\overrightarrow{F_{1}}+\overrightarrow{F_{2}}$}

$\odot$ Mettre les segments fléchés représentant $\overrightarrow{F_{1}}$ et $\overrightarrow{F_{2}}$ «bout à bout»: conserver les directions et les sens des vecteurs. L'extrémité du premier doit être à l'origine du second. On obtient le segment fléché représentant $\vec{F}$ en joignant l'origine du premier vecteur à l'extrémité du second.

○ Si on connaît l'échelle de représentation, la valeur de $\vec{F}$ se mesure directement sur la figure. Les relations trigonométriques permettent aussi de déterminer $\mathrm{F}$ par le calcul.

\section{Méthode analytique}

$\odot$ Choisir un repère orthonormé $(\hat{O}, \vec{i}, \vec{j})$. Deux axes suffisent lorsque les vecteurs sont dans un même plan.

$\odot$ Déterminer les coordonnées $\left(F_{1 x}, F_{1 y}\right)$ de $\overrightarrow{F_{1}}$ et $\left(F_{2 x}, F_{2 y}\right)$ de $\overrightarrow{F_{2}}$. Les coordonnées de $\vec{F}$ sont: $F_{x}=F_{1 x}+F_{2 x} ; F_{y}=F_{1 y}+F_{2 y}$

Encadré 1

Point-méthode

La méthode géométrique se présente comme une suite de règles d'actions avec un choix de vocabulaire volontairement très concret, comme si l'on voulait contrebalancer le langage hermétique des mathématiques. Tout ce qui est présenté ici fait normalement partie du bagage mathématique d'un élève de troisième, et est retravaillé en mathématiques en seconde. Les élèves connaissent également une autre méthode, dite règle du parallélogramme, pour calculer la somme de deux vecteurs, mais elle est totalement absente ici.

La méthode analytique est aussi connue des élèves depuis la classe de troisième en mathématiques. Le choix des notations est par contre particulier à l'enseignement de la physique et peut d'ailleurs rentrer en conflit avec l'habitude que les élèves peuvent avoir prise en mathématiques d'utiliser les indices 1 et 2 pour noter l'abscisse et l'ordonnée plutôt que les indices $x$ et $y$.

Voici un autre exemple (encadré 2) issu du manuel de physique de première $\mathrm{S}$ de Parisi (Curie, Deville, Guêtré et Heinrich, 2005). 
On considère un support plan incliné d'un angle $\alpha=20,0^{\circ}$ par rapport à l'horizontale. L'extrémité d'un ressort de raideur $\mathrm{k}=12,5 \mathrm{~N} \cdot \mathrm{m}^{-1}$ est fixé au support, tandis qu'à l'autre est accroché un palet autoporteur de masse $\mathrm{m}=410 \mathrm{~g}$ et de centre d'inertie $\mathrm{G}$. Le ressort est parallèle au support.

Un petit compresseur placé dans le palet envoie un jet d'air par un orifice situé au centre de la semelle du palet, afin de générer un coussin d'air entre le palet et le support.

Soit $\vec{R}$ la force exercée par le coussin d'air sur le palet.

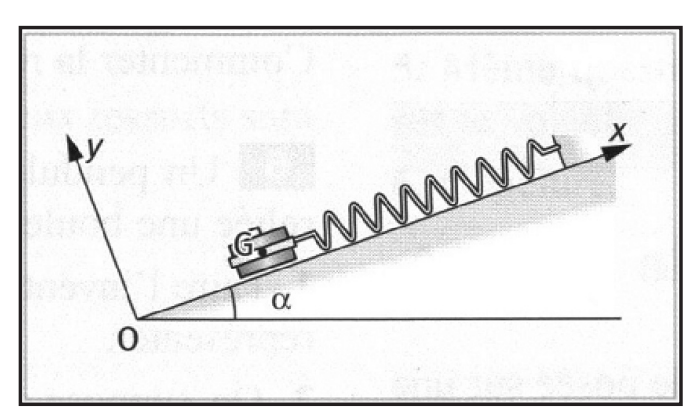

Quand l'ensemble est immobile, le ressort est allongé de $\Delta \mathrm{L}=110 \mathrm{rnm}$. On prendra g $=9,81 \mathrm{~N} \cdot \mathrm{kg}^{-1}$ pour la pesanteur au lieu de l'expérience.

1. Reproduire le schéma. Représenter la force de rappel $\vec{F}$ exercée par le ressort sur le palet. Calculer sa valeur $F$.

2. Même question pour le poids $\vec{P}$ du palet.

On considère le repère $0 x y$ dans le plan de figure. Les coordonnées du vecteur $\vec{R}$ sont notées $R_{x}$ et $R_{y}$.

3. Le palet étant immobile, indiquer en la justifiant la relation vectorielle vérifiée par $\vec{R}, \vec{F}$ et $\vec{P}$.

4. Calculer $R_{x}$ et $R_{y}$. Ajouter la force $\vec{R}$ sur le schéma, en choisissant un point d'application quelconque sur la semelle du palet.

5. Expliquer en conclusion le rôle du coussin d'air.

\section{Encadré 2}

Solide accroché à un ressort sur un plan incliné

La première remarque est que l'énoncé est très prolixe en explications sur la situation matérielle. On peut noter ici la différence avec les pseudo-exercices de physique présents dans les manuels de mathématiques. En ce sens, le dessin est typique de ce qui se fait en physique, avec un mélange, sur une même représentation d'objets "réalistes" (comme ici le ressort ou le palet) et de signes purement abstraits, comme les axes de coordonnées ou les forces. Par ailleurs, on notera que les lettres surmontées d'une flèche sont tantôt appelées vecteurs, tantôt appelées forces, alors qu'au début du cours les auteurs font la distinction entre la force et le vecteur force. Ce glissement sémantique est classique et symptomatique du rôle attendu des mathématiques. Enfin, la détermination de la force $\vec{R}$ est ramenée à un calcul numérique par la méthode imposée et détaillée pas à pas - à savoir projetée sur les axes -, alors qu'une caractérisation à l'aide de la trigonométrie aurait pu permettre une approche plus géométrique. En outre, si les connaissances et compétences mathématiques des élèves étaient prises en compte à la hauteur de ce qu'elles devraient être, les questions pourraient être moins nombreuses et la tâche plus ouverte, condition sine qua non pour faire vivre une interaction adéquate entre les deux disciplines. 


\section{Du côté des enseignants}

Afin de mieux cerner les rapports des enseignants des deux disciplines aux objets en jeu dans notre travail et aux liens entre les deux disciplines, nous avons finalement mené une enquête à l'aide de deux questionnaires destinés aux enseignants de sciences physiques d'une part, et aux enseignants de mathématiques d'autre part. Pour des questions pratiques, nous avons recueilli des données essentiellement au Sénégal, mais nos enquêtes précédentes (Ba, 2003) nous permettent de faire des rapprochements avec la situation en France.

Nous ne détaillerons pas ici nos analyses. De façon synthétique, nous donnons ci-dessous les trois aspects qui nous en semblent les plus saillants. Nous illustrons d'extraits des réponses obtenues qui montrent, malgré leur caractère individuel, des aspects institutionnels des problèmes que nous avons pu repérer:

1. Les enseignants avouent le plus souvent sans complexe ne pas connaître les programmes de l'autre discipline, voire retournent la question en disant que c'est à l'enseignant de l'autre discipline de connaître celui de la sienne.

C'est très net pour ce qui concerne les professeurs de mathématiques, mais voici deux réponses assez caractéristiques des réponses des professeurs de physique:

- C'est au professeur de mathématiques de connaître le programme de sciences physiques.

- Je ne vois pas l'intérêt pour le cours de physique et chimie.

2. Les collaborations entre enseignants des deux disciplines existent, sont même fréquentes, mais se cantonnent essentiellement à parler des élèves, à faire des ajustements curriculaires : ce n'est que très rarement qu'on y parle de contenu. Si les enseignants ont conscience qu'il y a un dysfonctionnement entre l'enseignement des deux disciplines, ils croient que sa source est essentiellement liée à des carences des élèves, ou des causes institutionnelles locales (mauvais ajustements curriculaires). Ils ne se rendent pas compte à quel point leur propre représentation de leur discipline et de ses liens avec l'autre peut être une barrière encore plus forte.

Voici une réponse d'un professeur de mathématiques :

- Nous discutons surtout du niveau des élèves, pour comparer ceux qui sont bien en maths avec ceux qui sont bien en physique, très souvent on retrouve les mêmes noms.

Et une autre d'un professeur de physique:

- On se voit de temps en temps car en mécanique (2S), nous avons besoin énormément des profs de maths (utilisation des vecteurs). En somme, nous demandons régulièrement au prof de maths de revenir sur telle ou telle notion pour nous faciliter la tâche. On constate ensemble que maths et physique sont très liées. Les élèves qui ont un bon niveau en maths l'ont aussi en physique.

3. Beaucoup d'enseignants de physique considèrent que le vecteur est un concept mathématique et que les difficultés des élèves avec les forces viennent avant tout de leurs carences en mathématiques. Les enseignants de mathématiques, eux, font le plus souvent allusion au rapport des vecteurs avec les forces, mais ne s'appuient pas sur des situations issues de la physique pour aborder des questions relatives aux vecteurs; ou s'ils le font, c'est, comme nous l'avons vu dans l'analyse des manuels, dans une sorte de parodie de modélisation. 
Voici par exemple deux extraits de réponses de professeurs de physique:

- Les élèves ont souvent des lacunes en maths; ils sont déroutés quand un prof de physique et chimie manipule des concepts mathématiques parfois inhérents à la description des phénomènes physiques. Le calcul vectoriel, les projections...

- Les notions mathématiques sont parfois mal maîtrisées. Les élèves peuvent avoir des difficultés pour faire le pont entre les connaissances maths et leur utilisation.

Et deux autres de professeurs de mathématiques :

- Je ne fais pas référence à la physique, car le cours risque d'être long et parfois même un peu complexe.

- Non. Je ne vois nullement l'utilité pour la compréhension des vecteurs; les élèves comprennent bien les vecteurs sans le support de la physique.

\section{Conclusion}

Le cloisonnement entre disciplines est-il une fatalité liée au fonctionnement même des institutions? Peut-on le gérer avec des injonctions dans les programmes officiels? Il est certain que cela devra au moins s'accompagner de dispositifs de formation qui mettent en rapport des enseignants des deux disciplines.

Mais au-delà de ce constat, on voit bien que c'est un manque de communication entre professions plus qu'entre individus qui est en jeu. Si du point de vue épistémologique, les liens entre vecteurs et grandeurs physiques vectorielles existent, l'histoire du système éducatif avec ses contraintes propres a en quelque sorte tout fait pour les rendre opaques par l'isolement des deux disciplines l'une de l'autre. Les enseignants des deux disciplines sont placés dans une logique qui empêche la collaboration là où elle devrait se faire et conduit à une méconnaissance des vrais enjeux. Les élèves, eux, s'habituent à un discours en porte-à-faux. Comment leur demander dans ces conditions de réinvestir à propos leurs connaissances de mathématiques en cours de physique et vice versa?

\section{Références}

Artaud, M. (1997). Introduction à l'approche écologique du didactique. L'écologie des organisations mathématiques et didactiques, In M. Bailleul et al. (dir.), Actes de la IXe École d'été de didactique des mathématiques, Houlgate, 1997, p. 101-139.

Artaud, M. (1999). Conditions et contraintes de l'existence des mathématiques dans l'enseignement général Permanences et évolutions, Petit x 50, 23-38.

Ba, C. (2003). Étude didactique de l'utilisation du vecteur en physique et des liens entre mouvement de translation et translation mathématique, mémoire de DEA, LIRDHIST, Université Claude Bernard, Lyon 1.

Ba, C. (2007). Étude épistémologique et didactique de l'utilisation des vecteurs en physique et en mathématiques. Thèse de doctorat - Université Claude Bernard - Lyon 1 et Université Cheikh Anta Diop - Dakar.

Ba, C. et Dorier, J-L. (2006). Aperçu historique de l'évolution de l'enseignement des vecteurs en France depuis la fin du XIX ${ }^{\mathrm{e}}$ siècle, l'Ouvert 113, 17-30.

Ba, C. et Dorier, J.-L. (2007). Liens entre mouvement de translation et translation mathématique : une proposition pour un cours intégrant physique et mathématiques, Repères IREM. 
Bittar, M. (1998). Les vecteurs dans l'enseignement secondaire - Aspects outil et objet dans les manuels - Étude de difficultés d'élèves dans deux environnements : papier-crayon et Cabri-géomètre II, Thèse de doctorat, Université Joseph Fourier - Grenoble 1.

Chevallard, Y. (1994). Les processus de transposition didactique et leur théorisation, 135-180, In G. Arsac (dir.) $L a$ transposition didactique à l'épreuve, Grenoble: La Pensée sauvage.

Crowe, M. J. (1985). A history of vector analysis: the evolution of the idea of a vectorial system (rééd.), Notre Dame University Press, New-York: Dover (1 $1^{\text {re }}$ éd. 1967).

Dorier, J.-L. (dir.) (1997). L'algèbre linéaire en question, collection Bibliothèque de Recherches en Didactique des Mathématiques, Grenoble: La Pensée Sauvage, éditions.

Dorier, J.-L. (2000). Recherches en histoire et en didactique des mathématiques sur l'algèbre linéaire - Perspective théorique sur leurs interactions, Cahier du laboratoire Leibniz $\mathrm{n}^{\circ}$ 12. Document téléaccessible à l'adresse $<\mathrm{http}: / /$ www-leibniz.imag.fr/LesCahiers/index.html>.

Flament, D. (1997). Le nombre, une hydre à $n$ visages. Entre nombres complexes et vecteurs. Paris: Éditions de la maison des sciences de l'Homme.

Flament, D. (2003). Histoire des nombres complexes. Entre algèbre et géométrie. Paris: CNRS Éditions.

Groupe de réflexion inter-associations de mathématiciens (GRIAM) (1998). Lycées, quels programmes pour quels objectifs. La Gazette des Mathématiciens 75, 51-63.

Lê Thi Hoai, C. (1997). Étude didactique et épistémologique sur l'enseignement du vecteur dans deux institutions: la classe de dixième au Vietnam et la classe de seconde en France. Thèse de doctorat, Université Joseph Fourier - Grenoble 1 et École Normale Supérieure de Vinh.

Lotz, E., Turner, J. et Misset, L. (2004). Mathématiques $2^{d e}$, collection Déclic, Paris : Hachette Éducation.

Ministère de l'Éducation nationale (1990). Programmes, mathématiques $2^{\text {de }}$, CNDP, Paris.

Ministère de l'Éducation. Programmes de mathématiques du moyen secondaire au Sénégal, CNM 2006.

Parisi, J.-M. (dir.), Curie, A., Deville, C., Guêtré, M. et Heinrich, A. (2005). Physique 1 ${ }^{r e}$ S, Collection Parisi, Paris: Belin.

Pressiat, A. (1999). Aspects épistémologiques et didactiques de la liaison "points-vecteurs ». Thèse de doctorat, Université Paris 7.

Tomasino A., Parent, C., Chappuis G., Meur, D. et Montangerand, M. (2001). Physique 1 ${ }^{r e}$ S, Collection Tomasino, Paris: Nathan. 